\title{
Research and application of new multimodal transport equipment-swap bodies in China
}

\author{
OU Chuan-jin ${ }^{*}$ and LI Bing-tao ${ }^{2}$ \\ ${ }^{1}$ Research Institute of Highway, Ministry of Transport, Beijing 100088, China, e-mail: \\ ${ }^{2}$ College of Mechanical Engineering, Jiamusi University, Jiamusi 154007, China
}

\begin{abstract}
Swap bodies emerged in Germany in the 1960s and is now a very mature and widely used product in Europe. Compared with Europe, the use of swap bodies in China started late. The application of swap bodies can effectively improve the technical level of the swap trailer transport equipment and accelerate the transformation of the modern road transport industry. This paper comprehensively introduces the size and transportation advantages of swap bodies, discusses the problems existing in the course of development, and expounds its prospect, providing a reference for the promotion and application of swap bodies in China.
\end{abstract}

\section{Introduction}

Since the reform and opening up, the road infrastructure in China has improved significantly, and the freight industry has made great progress. However, the road freight industry in China is still in an extensive development stage. The structure and types of freight vehicles are too miscellaneous, and the standardization degree of vehicles is too low. It has become a negative factor affecting the transformation and upgrading of the logistics industry, which has seriously restricted the smooth, efficient, safe, green and sustainable development of the transportation system. In order to promote the healthy development of the logistics industry, the State Council has successively issued the "Regulations for the Adjustment and Revitalization of the Logistics Industry" [1], "The Medium and Long-Term Plan for the Development of the Logistics Industry (2014-2020)" [2], and put forward the requirements for promoting the modernization of logistics technology and equipment. Developing joint distribution is an effective way to

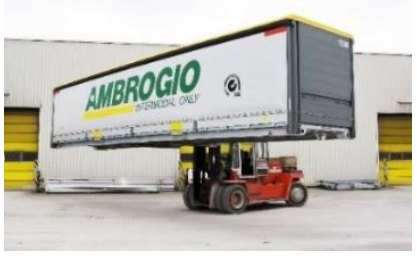

Figure 1. Class A swap body

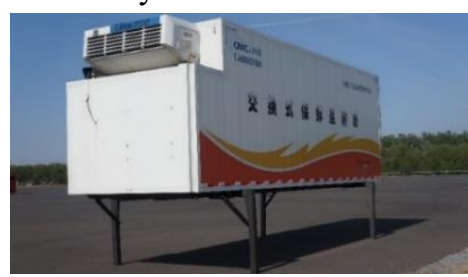

Figure 2. Class C swap body improve efficiency and rationally use resources to reduce urban traffic pressure and environmental pollution [3]. As a new mode of transport, the use of swap bodies for transportation is a convenient, flexible and efficient way of operation, with good social and economic benefits. Swap bodies will become an important delivery vehicle for multimodal transport in China [4].

\section{Classification of swap bodies}

The positioning size of bottom corners in the swap body is consistent with the corresponding ISO container. According to the positioning size of bottom corners, swap bodies can be divided into class A (Figure 1), class B and class $\mathrm{C}$ (Figure 2), which respectively correspond to $1 \mathrm{~A}$ (40 feet), 1B (30 feet) and 1C (20 feet) ISO container. According to the different structure forms of the box, the swap body can be divided into side curtain (Figure 3), tarpaulin (Figure 4), tank (Figure 5), drop side (Figure 6) and special purpose swap body.

Figure 1. Class A swap body

rigure 2. Class C swap body

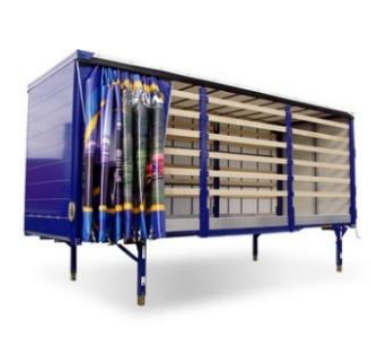

Figure 3. Sidecurtain swap body

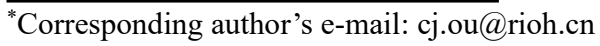




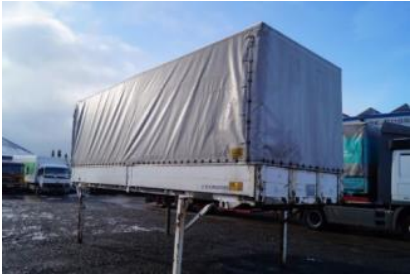

Figure 4. Tarpaulin swap body

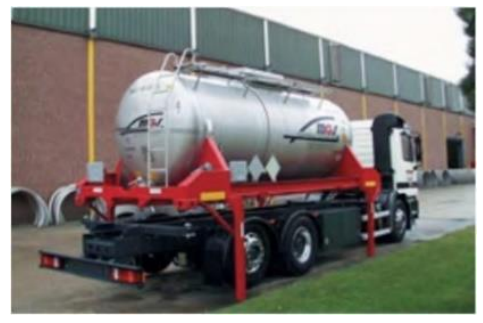

Figure 5. Tank swap body

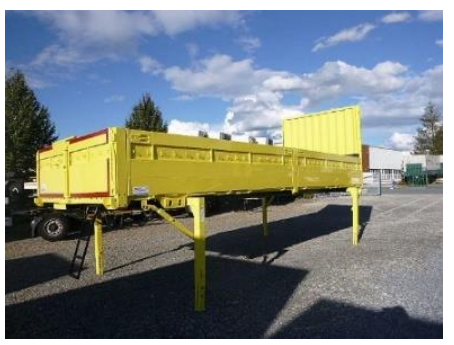

Figure 6. Drop side swap body
In EN 284:2006 [5], class C swap bodies are divided into two series - C745 and C782, according to the total length of the bodies, with length of $7450 \mathrm{~mm}$ and $7820 \mathrm{~mm}$ respectively, and the maximum total mass of $16 \mathrm{t}$. C715 swap bodies appeared in the 1992 version of EN 284, but in the next revision, it was cancelled. The length limit of road train in China is $20 \mathrm{~m}, 1.25 \mathrm{~m}$ longer than that of Europe's $18.75 \mathrm{~m}$, meanwhile, the length of the center-axle trailer is $8 \mathrm{~m}$. So, it is necessary to increase the C 800 swap body with a length of $8000 \mathrm{~mm}$ in China. If two C800 swap bodies are used, in order to ensure a sufficient safety distance between those swap bodies, the distance between the drawbar and the end of the towing vehicle should be adjusted to $1600 \mathrm{~mm}$, which can meet the limit of $20 \mathrm{~m}$ of the total length of the road train. At the same time, it ensures that no mechanical interference occurs. With the gradual maturity of China's swap bodies product technology, other series of swap bodies may appear in the future, and will be included in the standard as appropriate.

Among the three series of swap bodies, class C swap bodies are the most widely used in Europe and has the largest market share [4]. In main transport lines, two class C swap bodies can be loaded by the center-axle trailer combination. The center-axle trailer and one swap body can be thrown down to carry out urban logistics distribution by the truck. Meanwhile, because 20 feet is the basic module of logistics, class $\mathrm{C}$ swap bodies are also widely used in European rail transport system.

\section{Advantages of swap bodies transport}

Swap bodies transport has the following advantages:

(1) In road transport, when the vehicle combination arrives at the destination, the swap body on the vehicle combination can be unloaded and another swap body can be loaded without waiting for loading and unloading goods. In this way, the waiting time for operation can be reduced, the loading and unloading efficiency can be improved, and the use efficiency of the vehicle can be increased [6].

(2) Swap bodies can load and unload itself without lifting equipment or operating tools such as forklift, reducing the purchase cost of site equipment. In addition, a truck is generally equipped with 3 to 4 bodies, which can reduce the purchase cost of chassis, while reducing the number of drivers and reducing the operating cost.

(3) In the normal freight mode, the truck delivers goods to the destination and then returns empty. The swap body does not need to be fixed on the vehicle chassis all the time. After the swap body and the goods are unloaded from the vehicle, the empty truck can be returned. At this time, the empty weight of the truck is reduced, which can reduce the fuel consumption of the empty vehicle.

(4) Class C swap bodies have larger outer dimensions than the corresponding 20 -foot container, and the pallets number is also larger. For example, C782 swap body can load $181200 \mathrm{~mm} \times 800 \mathrm{~mm}$ pallets or 14 $1200 \mathrm{~mm} \times 1000 \mathrm{~mm}$ pallets, while $1 \mathrm{C}$ container can only load $111200 \mathrm{~mm} \times 800 \mathrm{~mm}$ pallets or $91200 \mathrm{~mm} \times 1000 \mathrm{~mm}$ pallets, leading to loading capacity increase of $63.6 \%$ or $55.6 \%$ respectively.

\section{Problems in the development of swap bodies}

In recent years, with the transformation of express logistics enterprises, swap bodies have been recognized and accepted by more and more enterprises. Large logistics companies in China have a strong interest in this new equipment. Despite this, there are still great difficulties in the promotion of swap bodies in China.

(1) The "small, scattered, weak" condition of the freight market limits the promotion of swap bodies. In recent years, road transport industry in China has developed rapidly, but for a long time, the development has been too extensive, lacking intensive and networked transport organizations. The premise of using swap bodies transport is to have a stable and sufficient business volume. At present, China's logistics market is still in the primary development stage. Due to the low access requirement for freight, it is still dominated by small and medium-sized logistics enterprises and retail investors. They are still in a state of seeking survival with small business volume. It is difficult and uneconomical to carry out swap bodies transport.

(2) Illegal modification of freight vehicles and overlimit phenomena have destroyed the market base for the development of swap bodies. Since 2016, various localities and relevant departments have organized special actions to rectify the illegal over-limit and overload of road trucks, and achieved positive results. However, in some areas, the illegal modification of trucks and overloading of transport is particularly prominent, causing safety hazards, leading to some major accidents. The survey found that the breast board height of the dump truck and the drop-side truck is generally increased, with a volume increased of more than $30 \%$; the van is painted 
with different colors, identification marks and modified container fixing devices to disguise as an international standard container, thereby obtaining highway tolls in the form of container transport. Illegal modification and overloading have destroyed the fair market environment on which swap bodies are based, limiting the development of new structures, new materials and new technologies.

(3) The high update cost of the transport mode limits the development of swap bodies. Swap bodies transport has higher requirements for hardware and software, such as vehicles, loading and unloading equipment and sites. The most important thing among them is the operation site. In recent years, the land cost has risen sharply, and the land acquisition is very difficult. If there is no suitable operation sites, swap bodies transport cannot develop. According to the current development model of logistics enterprises in China, if the use of new transport mode costs more capital, it is not meet the short-term development goals of transport enterprises.

\section{Swap bodies development application prospects}

The application of swap bodies will promote swap trailer transport and multimodal transport, which can reduce the number of heavy trucks on the highway by about $10 \%$, increase the loading capacity by about $60 \%$, reduce the transport cost by about $30 \%$, and reduce the fuel consumption and greenhouse gas emission of the unit loading capacity - transport distance $(\mathrm{t}-\mathrm{km})$ by about $15 \%$.

At present, with the increase of swap bodies truck orders in express logistics company, many truck manufacturers and special purpose vehicle enterprises have also launched a lot of corresponding products. Sinotruk Qingdao Heavy Industry Co., Ltd., CIMC Vehicle (Shandong) Co., LTD., and other enterprises have developed transport trucks for loading swap bodies and central-axle trailer, but just small batch production and application. In addition, domestic vehicle enterprises have mastered the standards and core technologies of European swap bodies, and began mass production, and some export to Europe, laying a good technical foundation for the promotion and application in China [7].

According to the current situation and requirements of the transformation and development of transport and standardization reform, in order to meet the urgent needs of the modernization of transport equipment, meanwhile, to standardize the healthy development of swap bodies transport industry, the following work needs to be carried out:

(1) In order to standardize the production, use and management of the swap bodies, it is necessary to perfect the standard system of swap bodies, formulate and publish a series of standards as soon as possible. At the same time, in order to reduce the number of standards, avoid duplication of content, ensure the integrity and system of standard content, and facilitate the use of standards, the product requirements and test methods of swap bodies should be integrated.

(2) With the development of urban freight, small sway bodies have gradually emerged, as shown in Figure 7.
Swap bodies can be changed into various styles according to different purposes. The biggest advantage of them is that they are more widely used and can be placed in any open space near the supermarket or shop, not subject to the restrictions of the site. Thus, it can alleviate urban road traffic congestion. Technical staff should actively track the development and application of new swap bodies product and technology, including some small swap bodies product demand, use characteristics, technical requirements, etc.

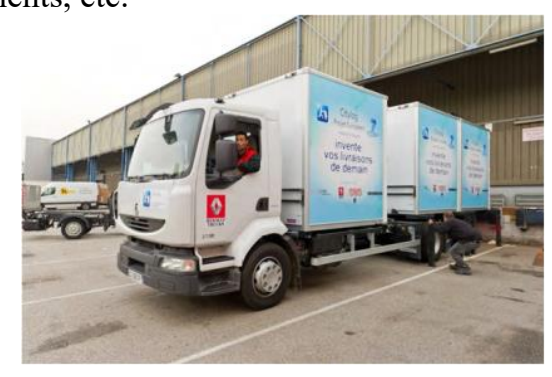

Figure 7. Small swap body

\section{Conclusion}

China's road freight industry is still in the stage of extensive development, the vehicle standardization degree is too low, and illegal modification of freight vehicles and overload transport still exit, which has restricted the sustainable development of road transport. As a new transport equipment, the swap body for transport can reduce the weight of empty vehicles, save manufacturing materials, reduce fuel costs, achieve swap trailer transport, effectively improve the efficiency of vehicle use, and promote the standardization of cargo transport. Swap bodies transport is a convenient, flexible and efficient operation mode, with good social and economic benefits. It is possible and necessary to popularize and apply this technology, which will bring about a fundamental change in the mode of transport in China and have a far-reaching impact.

\section{Acknowledgements}

This work is sponsored by National Key Research and Development Project "Research on Logistics Basic Modulus and Application Standards"(No. 2017YFF0208701).

\section{REFERENCES}

1. State Council of China (2009) Regulations for the Adjustment and Revitalization of the Logistics Industry. http://www.gov.cn/zwgk/200903/13/content_1259194.htm

2. State Council of China (2014) The Medium and Long-Term Plan for the Development of the Logistics Industry (2014-2020). http://www.gov.cn/zhengce/content/201410/04/content_9120.htm 
3. Elvezia M., Alessandro F. (2015) A new urban freight distribution scheme and an optimization methodology for reducing its overall cost. European Transport Research Review, 2015, $7(1): 1-14$.

4. Ji P. (2016) More efficient than swap trailer, swap body is gradually becoming a new favourite. Special purpose vehicle, 2016 (1), 54-57.

5. EN 284 (2006) Swap bodies - Non-stackable swap bodies of class C - Dimensions and general requirements. Brussels, CEN.

6. Shao J., Xu J., Sun Y. (2015) Study on Urban Joint Distribution Mode Based on Swap Body Technology. Logistics Technology, 2015, 34:1113.

7. Zheng Shu. (2017). "Twists and turns, the rise and prospect of swap bodies in China". Special purpose vehicle, 2017 (6), 33-36. 